\title{
La bibliothèque de l'École des mines, lieu de savoir et lieu de mémoire pour les ingénieurs
}

Isabelle Laboulais

\section{(2) OpenEdition}

12 Journals

Édition électronique

URL : https://journals.openedition.org/rbnu/1627

DOI : $10.4000 /$ rbnu. 1627

ISSN : 2679-6104

Éditeur

Bibliothèque nationale et universitaire de Strasbourg

Édition imprimée

Date de publication : 1 novembre 2014

Pagination : 44-55

ISBN : 9782859230548

ISSN : 2109-2761

Référence électronique

Isabelle Laboulais, "La bibliothèque de l'École des mines, lieu de savoir et lieu de mémoire pour les ingénieurs », La Revue de la BNU [En ligne], 10 | 2014, mis en ligne le 01 novembre 2014, consulté le 17 mai 2021. URL : http://journals.openedition.org/rbnu/1627 ; DOI : https://doi.org/10.4000/rbnu.1627

\section{(c) (i) (-)}

La Revue de la BNU est mise à disposition selon les termes de la Licence Creative Commons Attribution - Pas d'Utilisation Commerciale - Partage dans les Mêmes Conditions 4.0 International. 


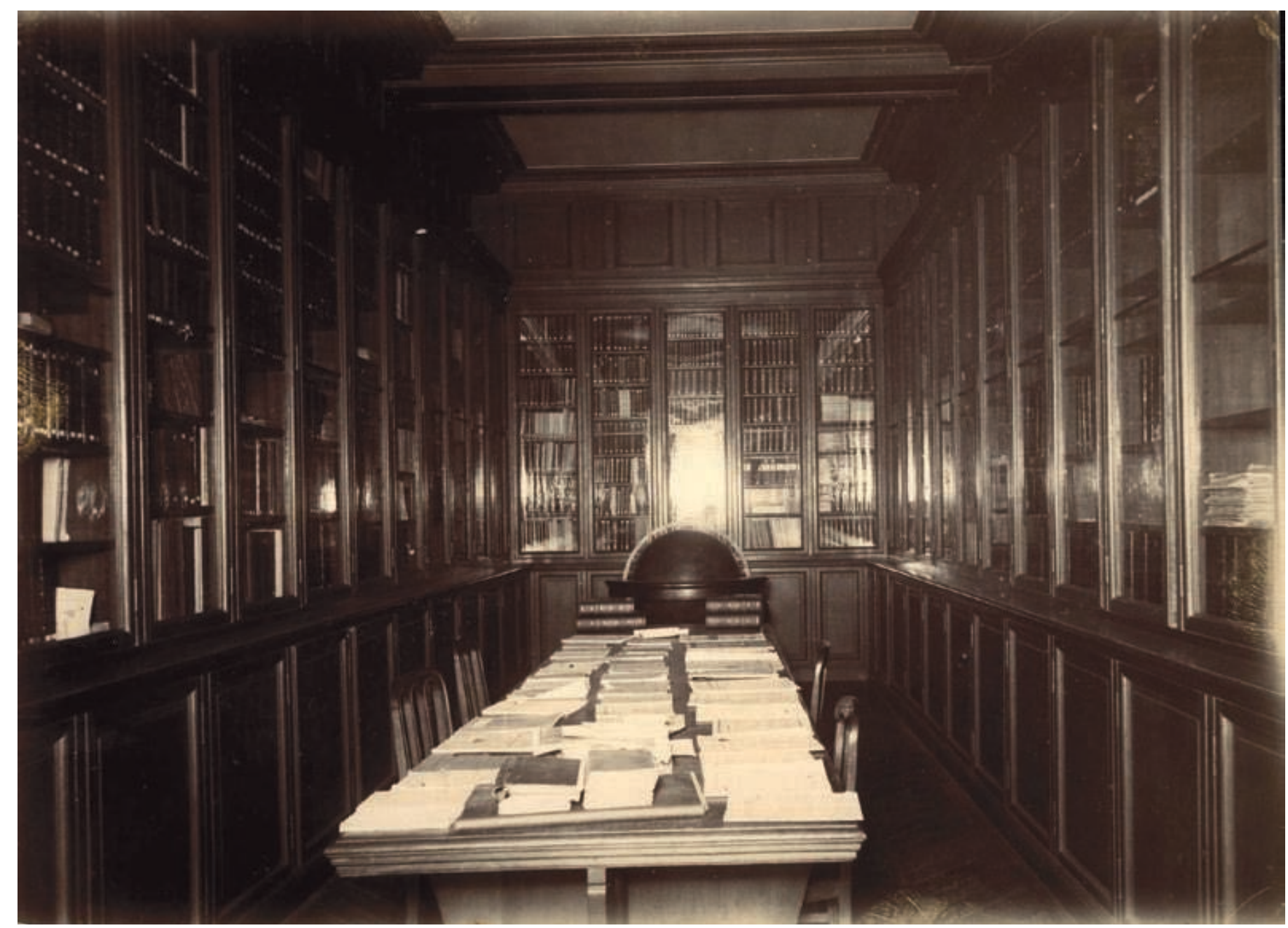




\section{La bibliothèque de l'École des mines, lieu de savoir et lieu de mémoire pour les ingénieurs}

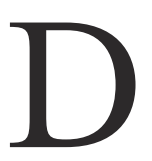

epuis la fin de l'Ancien Régime, l'École chargée de la formation des membres du corps des Mines a investi successivement trois hôtels parisiens - à partir de 1778, l'hôtel de la Monnaie, quai Conti, à partir de 1794, l'hôtel de Mouchy, rue de l'Université, enfin, à partir de 1815 , l'hôtel de Vendôme, rue d'Enfer - ; entre 1802 et 1814, elle a été temporairement installée en Savoie, à proximité de la mine de Pesey. C'est à partir de la Révolution que le souci de constituer, au sein de l'École des mines, une collection publique de livres utiles à la formation des ingénieurs s'est affirmé et a suscité une réflexion sur la forme que devait prendre le lieu propre à la conservation de ces ouvrages et à leur consultation ${ }^{1}$. Les livres utilisés par les ingénieurs des Mines, les lieux qui leur sont consacrés, les pratiques qui s'y déploient regardent la culture professionnelle et l'identité de ce corps d'ingénieurs civils, autant que l'histoire institutionnelle des bibliothèques.

La bibliothèque de l'École des mines permet de questionner le rapport d'un corps d'ingénieurs civils aux livres en général, aux livres de science en particulier. Depuis la création de la Maison des mines et la nomination des premiers ingénieurs en 1794, la bibliothèque joue un rôle central dans le fonctionnement de l'École, non seulement parce qu'elle abrite les ouvrages dont les professeurs et les élèves ont besoin, mais aussi parce que les livres mettent en scène une sorte de communauté de savoir à laquelle accède tout nouvel élève, puis à laquelle est agrégé tout nouvel ingénieur. La genèse des collections de livres laisse entrevoir les configurations de savoirs qui jalonnent l'histoire du corps des Mines. Les pratiques à l'œuvre au sein de la bibliothèque de l'École permettent de retrouver les deux formes que peut prendre une communauté : le cercle et la lignée ${ }^{2}$. Espace où se déploient des pratiques collectives de travail intellectuel, la bibliothèque suscite des signes de reconnaissance et d'appartenance. Les dons de livres effectués par les descendants de certains ingénieurs des Mines à la bibliothèque de l'École témoignent quant à eux d'un lien identitaire très marqué avec le corps et expriment la volonté de l'inscrire dans la durée. Ils peuvent être examinés comme des " rites d'institution ", c'est-à-dire comme une procédure " visant à produire un groupe séparé et sacré ${ }^{3}$. On peut se demander si l'on ne touche pas là " l'aura symbolique » dont Pierre Nora juge l'intervention nécessaire pour donner à un lieu d'apparence purement matériel, comme un dépôt d'archives, le statut de lieu de mémoire ${ }^{4}$.

\section{Créer une « bibliothèque nationale des Mines " : la construction et l'essor d'une collection spécialisée}

Les sources qui permettent de suivre la constitution de cette collection sont éparses et fragmentaires. Les listes d'achats n'ont que très rarement été conservées, de même que les budgets annuels de la bibliothèque. Les catalogues constituent une source importante pour reconstituer le contenu des collections, même si tous n'ont pas été conservés ${ }^{5}$. En dépit des limites qu'impose la documentation, il est possible de saisir l'ampleur des collections, ainsi que la manière dont les grands domaines de savoirs y sont représentés et s'y côtoient. 
Comme pour la bibliothèque des Ponts et Chaussées ou celle de l'École polytechnique, les confiscations révolutionnaires, qui constituent l'origine principale des premiers ouvrages rassemblés dans la bibliothèque aménagée rue de l'Université ${ }^{6}$, ont contrarié les volontés de spécialisation. En dépit du souhait explicite de créer une " bibliothèque nationale des mines ${ }^{7}$, les premiers ouvrages à entrer dans les collections furent des volumes de l'Encyclopédie méthodique, des ouvrages de physique et de chimie, quelques autres d'histoire naturelle ${ }^{8}$. Rares étaient les volumes qui concernaient la " science des mines ", si bien qu'à la fin de l'automne 1795, alors que les cours étaient sur le point de commencer, aucun des livres nécessaires aux professeurs n'était disponible ${ }^{9}$. L'enseignement constituait l'une des raisons d'être de cette bibliothèque et la collection devait disposer des ouvrages de référence pour la discipline. Pour y parvenir, le Conseil des mines demanda en 1796 que son bibliothécaire, Clouet, soit autorisé à prendre connaissance « des livres relatifs à la science des mines qui peuvent exister dans les dépôts ${ }^{10}$. Clouet retint 630 titres qu'il répertoria dans un registre en les classant par rubrique et par ordre alphabétique d'auteurs ${ }^{11}$. À l'exception des usuels, seuls des ouvrages de science furent retenus. Les équilibres restent pourtant surprenants : la minéralogie $(15 \%)$ arrive loin derrière les mathématiques et les voyages (chacun constituant $28 \%$ de l'ensemble). La plupart de ces ouvrages étaient publiés à Paris, quelques-uns à Londres ou dans le monde germanique, mais cette collection restait assez généraliste. Pour parvenir à constituer un fonds spécialisé, l'appropriation de collections spécifiques sembla plus prometteuse. La bibliothèque de P.-F. de Dietrich le montre bien. Composée de 1030 volumes dont la majeure partie était en allemand, cette collection fut d'abord confisquée, puis restituée à la famille, enfin rachetée par le ministère de l'Intérieur aux héritiers de Dietrich. Grâce à cette collection, le Conseil des mines put revendiquer la spécificité de sa bibliothèque au sein de l'espace parisien. En 1799, elle rassemblait environ 1800 monographies et plus de 20 titres de périodiques. Si le nombre de volumes n'était pas considérable, la recherche de spécialisation restait néanmoins perceptible. L'ancrage de la "science des mines " du côté de l'histoire naturelle et de la physique s'impose. Contrairement aux usages que l'on peut observer dans l'espace germanique, le droit et l'économie n'étaient pas représentés. Cette collection confirme l'ambiguïté de ce lieu, tiraillé entre les savoirs savants qui fondaient la culture du corps des Mines et les savoirs de gouvernement qui constituaient son champ d'intervention ${ }^{12}$.
En 1802, lors de l'installation de l'École sur la mine de Pesey, la nécessité de disposer d'une bibliothèque fut réaffirmée. Les collections rassemblées à Paris restèrent dans les locaux de la Maison des mines. En 1803, le préfet, le sous-préfet et le maire de Moutiers proposèrent que les livres de la bibliothèque de la ville soient réquisitionnés pour constituer le fonds de la bibliothèque spécialisée de l'École de Pesey ${ }^{13}$. Une bibliothèque généraliste qui ne disposait d'aucun équipement, ni d'aucun personnel, fut donc réunie à une bibliothèque spécialisée qui ne possédait aucun livre. La collection était alors composée de 1570 volumes provenant de la bibliothèque municipale de Moutiers et de 208 autres appartenant à l'École des mines ${ }^{14}$. En 1808 , cette collection, destinée aux élèves et aux professeurs, fut installée dans l'ancien séminaire de Moutiers, à une vingtaine de kilomètres de Pesey. C'est là que les cours théoriques étaient dispensés et que les spécimens des collections minéralogiques étaient disposés. Lorsque la Savoie revint au Piémont, l'École pratique dut quitter Pesey et Moutiers. Les livres, peu nombreux et trop peu spécialisés aux yeux des professeurs et des élèves, furent rapatriés à Paris.

En 1816, une ordonnance royale officialisa le retour de l'École des mines à Paris. La bibliothèque aménagée dans les locaux de l'École ne chercha plus à apparaître comme un lieu de savoir au sein de l'espace savant parisien, mais plutôt comme un outil de travail adapté aux besoins des professeurs et des élèves ; les collections d'imprimés constituées au $19^{e}$ siècle en témoignent. L'École procéda à l'achat de grandes collections de périodiques et d'ouvrages utiles aux professeurs comme aux élèves, un fonds technique orienté vers les mines et la métallurgie fut constitué. Les échanges perdurèrent, notamment pour les périodiques. Comme c'était déjà le cas pendant la Révolution et l'Empire avec le Journal des mines, les numéros des Annales des mines étaient échangés avec d'autres périodiques publiés ailleurs en France ou à l'étranger, et consacrés à l'exploitation des mines et à la géologie. La bibliothèque recevait également des dons venus d'autres institutions publiques (l'École des ponts, le ministère du Commerce à l'occasion de l'exposition universelle de 1883). La liste des ouvrages qui, à ces occasions, arrivaient dans les collections de l'École des mines témoigne de l'identité acquise par cette bibliothèque. On y retrouve une forte emprise de la culture technique, de la science de l'ingénieur. 


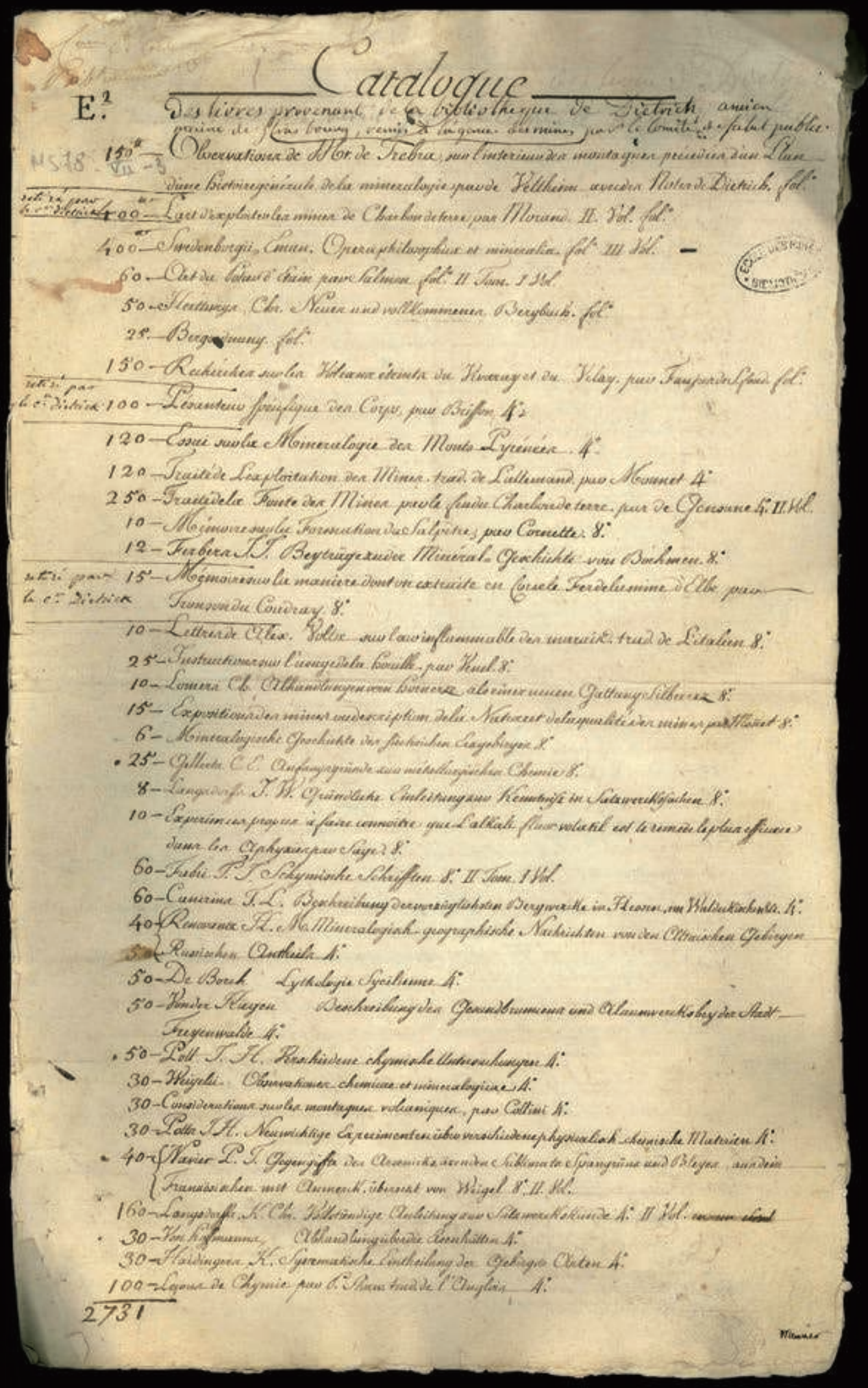



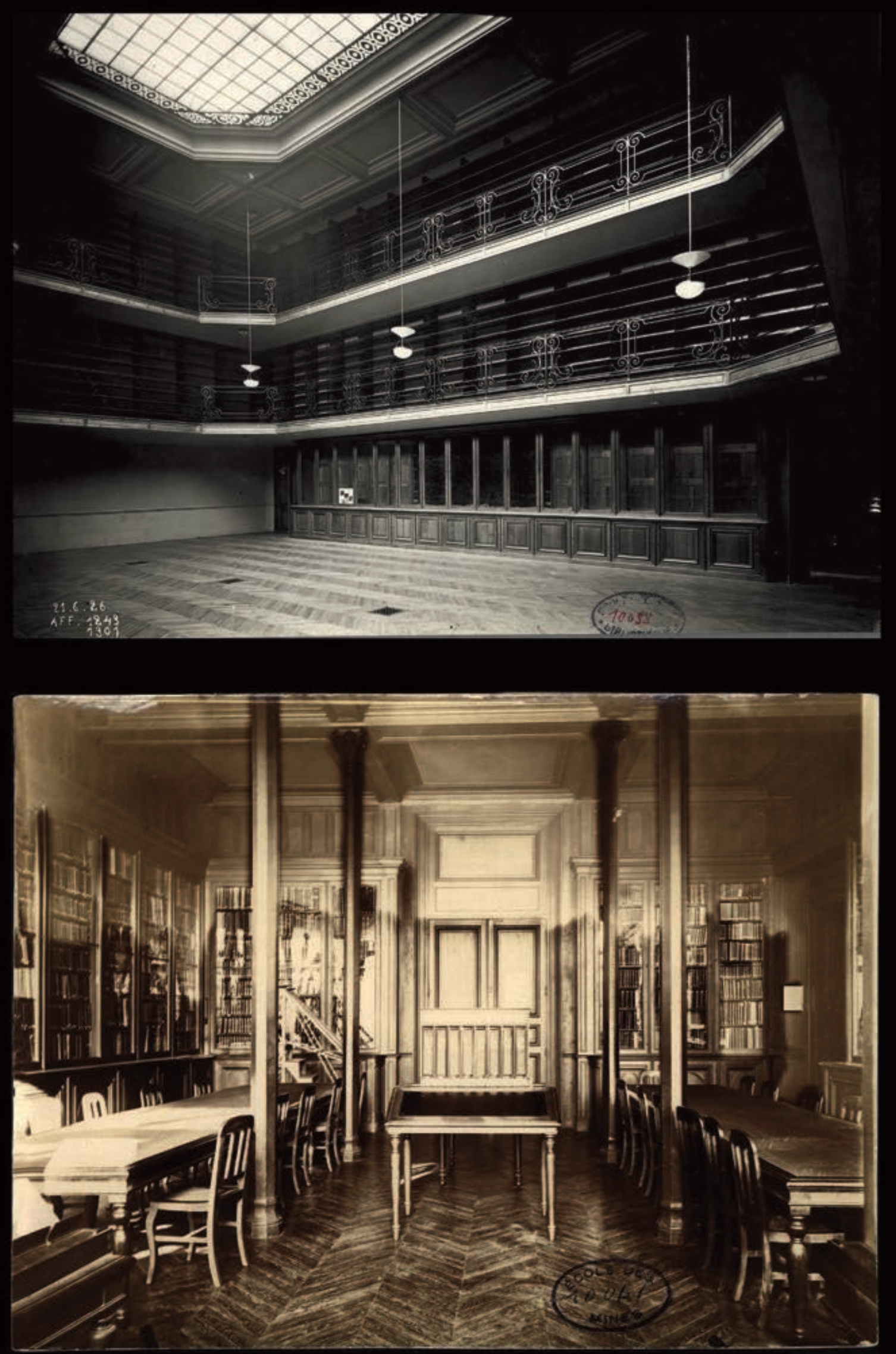

(Haut) Grande salle de lecture de la bibliothèque de l'École des mines (vers 1925; coll. bibliothèque de l'École des mines de Paris) (Bas) Nouvelle salle des périodiques de la bibliothèque de l'École des mines, inaugurée en 1926 (coll. bibliothèque de l'École des mines de Paris) 
Passée la difficile spécialisation des premiers temps, les collections de la bibliothèque de l'École des mines ont suivi l'élargissement des disciplines enseignées. L'emprise de la minéralogie et plus encore de la géologie est restée forte ; cependant la statistique, l'économie, le droit ou la sociologie se sont progressivement immiscés dans les collections. Les acquisitions, les échanges et les dons ont assuré leur accroissement régulier au cours des $19^{\mathrm{e}}$ et $20^{\mathrm{e}}$ siècles. En 1904, la bibliothèque de l'École des mines conservait environ 42000 volumes ${ }^{15}$. En 1953, elle en contenait 250000 , et plus de 2000 collections de revues. Aujourd'hui la bibliothèque possède 200 ooo documents sur support papier dont 40 ooo antérieurs à 1900, 62000 publiés entre 1900 et 1975 et 81500 postérieurs à 1975 .

\section{Aménager un lieu de travail : les enjeux d'une salle de lecture}

L'existence d'une collection de livres constituée par et pour une grande école ne suffit pas à mettre au jour les usages que les professeurs et les élèves ont eus de ces imprimés. Il faut les resituer dans l'espace qui leur était consacré. L'existence d'un local dévolu au rangement des volumes n'a pas la même portée que la création d'une salle de lecture. L'existence d'un personnel spécifiquement assigné à cet espace et les fonctions qui lui sont confiées apparaissent aussi comme un paramètre déterminant pour saisir les pratiques. Les sources sont parfois lacunaires (les plans, par exemple, n'ont pas toujours été conservés), de sorte qu'il est difficile de savoir à partir de quand la bibliothèque a impliqué l'aménagement d'un espace propre à la consultation des ouvrages du fonds. D'autres documents - des lettres notamment - font allusion aux pratiques à l'œuvre en son sein.

Pendant la Révolution et l'Empire, la bibliothèque créée au sein de la Maison des mines fut installée au premier étage du bâtiment de la rue de l'Université, et elle y resta jusqu'au déménagement de $1814^{16}$. Aucune donnée ne permet de connaître sa superficie, ni son ameublement. Certains témoignages suggèrent pourtant que des tables de travail étaient disposées dans cette pièce. Elle était ouverte " tous les jours impairs depuis 10 heures jusqu'à 4 heures $"{ }^{17}$. Les registres de prêts montrent qu'elle était fréquemment utilisée, tant par les membres du Conseil et de l'inspection des mines que par certains chefs de bureau, ainsi que par les élèves ${ }^{18}$. Ce lieu était conçu pour faciliter le travail des membres du corps ainsi que celui des employés de bureau et celui des savants attachés à cette institution. Plus qu'un espace de travail, la bibliothèque apparaît comme un espace pour le travail. En 1802, lorsque l'École pratique des mines fut créée en Savoie, plus aucun cours n'était dispensé à Paris ; cependant la bibliothèque réunie au premier étage de la Maison des mines y fut maintenue. Ces ensembles documentaires furent utilisés par les employés de bureau et les ingénieurs qui travaillaient dans les locaux parisiens. Lorsqu'en 1814 l'administration des mines dut quitter la rue de l'Université, les collections furent déplacées à deux reprises en moins d'un an, d'abord vers le palais du Petit Luxembourg où elles se trouvèrent " entassées confusément et sans ordre ${ }^{19}$ " puis, quelques mois plus tard, à l'hôtel de Vendôme où la bibliothèque de l'École des mines se trouve encore aujourd'hui ${ }^{20}$.

En 1816, trois membres du corps des Mines, Baillet, Duhamel et Lefroy, furent chargés par le conseil de l'École de formuler quelques propositions sur la meilleure manière d'aménager dans l'hôtel de Vendôme les espaces nécessaires au bon fonctionnement de l'établissement. Dès 1816 , la bibliothèque prit possession de trois salles au rezde-chaussée. Trois fonds furent identifiés : celui des livres destinés aux ingénieurs et aux élèves, celui conservant les plans et dessins, celui regroupant les ouvrages et journaux; mais il apparut très vite que la place manquait ${ }^{21}$. En 1819, la bibliothèque fut dotée d'une pièce supplémentaire, et l'on put songer à mettre un peu d'ordre dans les collections, notamment celles des statistiques départementales. En 1837, l'État se porta acquéreur du bâtiment, ce qui permit d'entreprendre des travaux de grande ampleur pour adapter cet ancien hôtel particulier aux besoins d'une école. Le corps central du bâtiment fut préservé et complété par deux ailes achevées en 1852 . Toutefois, dès 1860 , le percement du boulevard Saint-Michel obligea à détruire une partie des nouveaux bâtiments. Une nouvelle série de travaux intervint à partir de 1866 . La bibliothèque fut maintenue au rez-de-chaussée de l'École. Elle disposa d'une salle de lecture qui était parfois utilisée pour les réunions du Conseil général des mines, ainsi que d'une salle des périodiques (ill. p. 44). Mais les espaces dévolus à la bibliothèque furent de nouveau modifiés à partir de 1924. La grande salle de lecture fut construite sur une galerie réunissant deux bâtiments (ill. p. 48). Cette nouvelle bibliothèque, inaugurée en 1926 par le maréchal Foch, disposait de trois salles : une salle des périodiques où se trouvait le bureau de prêt (ill. p. 48), une salle des catalogues, enfin une salle de lecture. Les derniers travaux de grande ampleur furent entrepris en 1992 lorsque des magasins furent construits au sous-sol, afin d'assurer des conditions de conservation meilleures que celles offertes par les mansardes. 
UNIVERSITE DE PARIS

INSTITUT D'HISTOIRE

DES SCIENCES

ET DES TECHNIQUES

13, RUE DU FOUR, 13

PARIS-VI

TEL. : ODEON 60.36
LE $20^{\circ} \operatorname{tac} \cos \theta 4$

terosicue le Beceelceer,

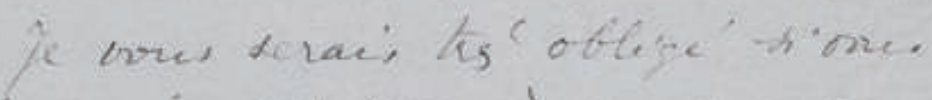

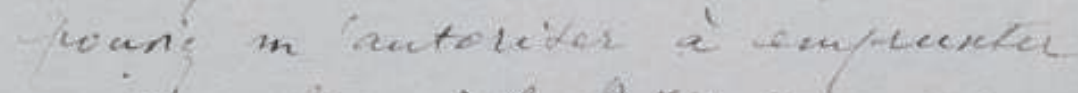

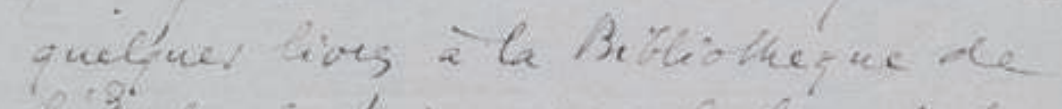

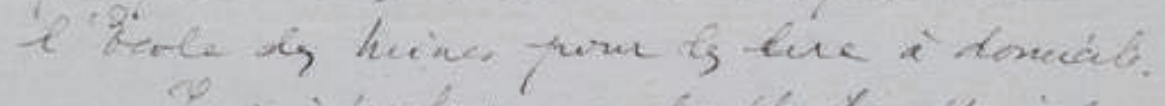

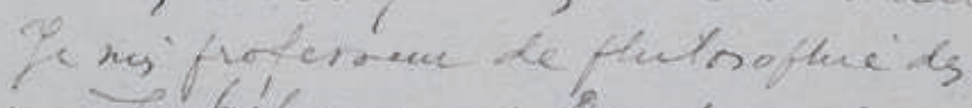

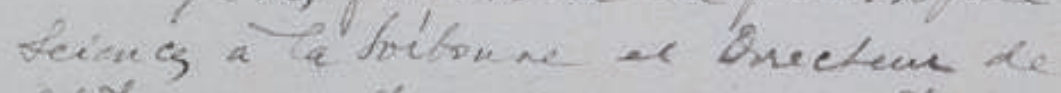

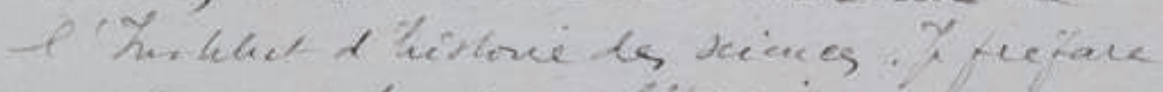

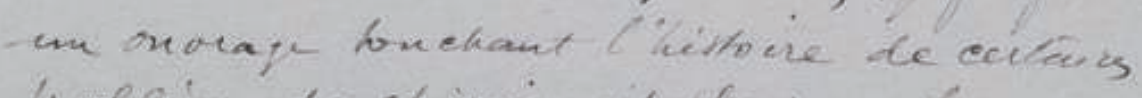

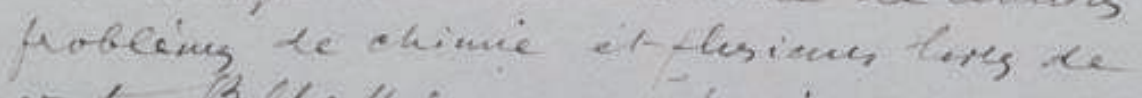

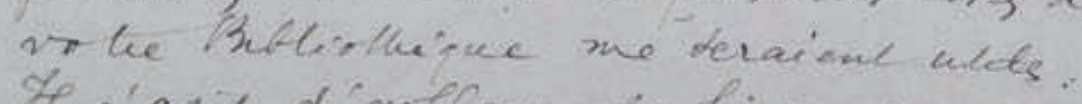

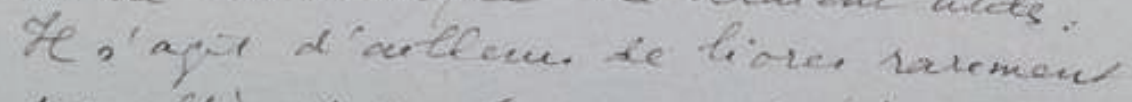
tow chlsi te

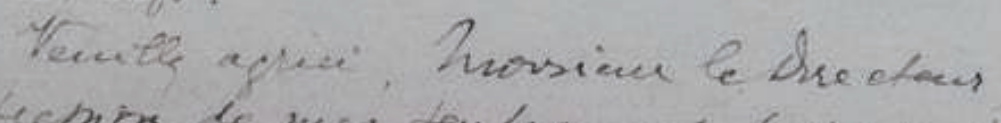

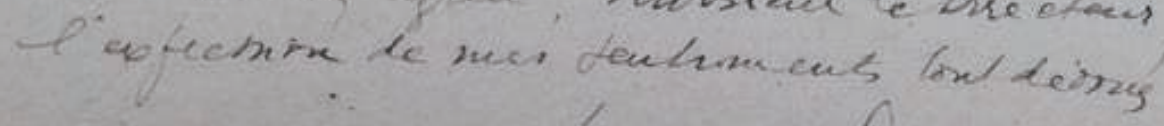

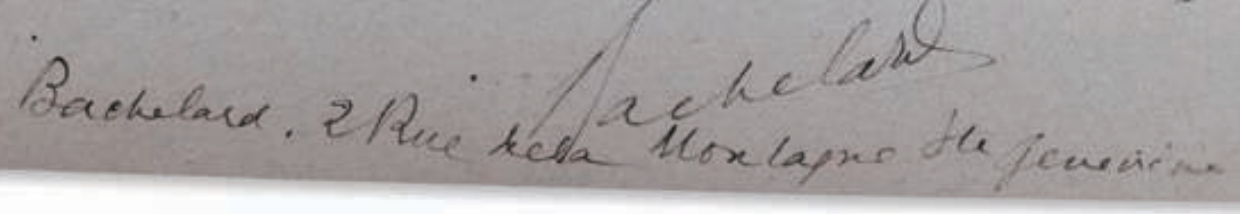

Lettre de Gaston Bachelard au directeur de la bibliothèque, sollicitant l'autorisation (qui lui fut refusée) d'emprunter des ouvrages du fonds (coll. bibliothèque de l'École des mines de Paris)

50 
Aucun document ne permet de suivre le circuit des emprunts existant aux $19^{\mathrm{e}}$ et $2 \mathrm{O}^{\mathrm{e}}$ siècles, de retrouver les usages de certains professeurs, ou ceux des élèves. Les règlements successifs témoignent en revanche de l'élargissement des horaires d'ouverture et de l'évolution des règles qui régissaient le fonctionnement des prêts. En 1912, les professeurs, les chefs de travaux pratiques et les préparateurs pouvaient emprunter des ouvrages pour un mois ; les élèves devaient se contenter d'une journée, ou huit jours à titre exceptionnel ; quant aux anciens élèves, ils pouvaient emprunter des ouvrages " au titre de faveur ". En 1921, un nouveau règlement interdit que les livres sortent de la bibliothèque ; il imposait aux lecteurs de ranger eux-mêmes les volumes qu'ils avaient consultés. Les prêts reprirent dans les années 1940, mais ils restaient toutefois strictement réservés aux membres du corps. Il n'était vraisemblablement pas dans les habitudes du directeur de tolérer des exceptions puisque même la demande de Gaston Bachelard (ill. p. 5o) qui, en 1944, espérait emprunter des ouvrages de chimie, essuya un refus catégorique. La fréquentation de la salle de lecture progressa : le conservateur indique 12 ooo lecteurs en salle de travail pour 1953, et 6 ooo volumes prêtés à des personnes extérieures au corps, ce qui suggèrel'attractivité grandissante de cette bibliothèque.

\section{Les travaux de catalogage et le signalement des collections}

Au cours du $19^{e}$ siècle, au moment où les collections bénéficièrent d'un accroissement régulier et furent stockées dans des espaces stabilisés, la tenue de registres et la réalisation de catalogues se généralisèrent, faisant écho à l'augmentation du nombre de lecteurs. À partir des années 1840, les titres des ouvrages qui entraient dans les collections de la bibliothèque furent consignés dans un registre. C'est vraisemblablement à la même période qu'un premier catalogue sur fiches fut constitué (ill. p. 53), les monographies y étant répertoriées par ordre alphabétique d'auteurs et les périodiques présentés, dans une boîte particulière, par ordre alphabétique de titres.

En 1899-1900, quelques années après l'École polytechnique ${ }^{22}$ et l'École des ponts et chaussées ${ }^{23}$, le premier catalogue imprimé de la bibliothèque de l'École des mines fut publié en deux volumes. Il s'agit d'un catalogue systématique, complété par un index par nom d'auteurs. Il recensait environ 30 ooo titres répartis par disciplines ${ }^{24}$. Le premier volume était consacré aux sciences exactes (mathématiques, physique, minéralogie et géologie qui représente un quart du fonds), le second aux sciences appliquées (métallurgie, machines, chemins de fer, statistique, expositions industrielles, commerce, c'est-àdire une rubrique très englobante qui intègre l'économie politique, la sociologie, les finances et le budget) ${ }^{25}$. C'est une commission de professeurs qui établit le classement pragmatique retenu dans le catalogue ${ }^{26}$. Il devait fournir aux enseignants et aux élèves un outil pour trouver les documents dont ils avaient besoin, en fonction de leurs connaissances scientifiques et de leur façon d'appréhender les disciplines. Il devait aussi faire connaître les fonds de l'École des mines, comme en témoigne la liste des institutions ou des personnes auxquelles les deux volumes du premier catalogue imprimé de l'École ont été envoyés (ministères, Sénat, Chambre des députés, Conseil d'État, membres du corps des Ponts et Chaussées, membres du corps des Mines, correspondants étrangers de l'École des mines).

En 1943, la version manuscrite de l'ancien catalogue méthodique, qui était posée sur les tables de la salle de lecture, fut convertie sur des fiches cartonnées de 15 sur $10 \mathrm{~cm}$. Cette entreprise suggère que les fonds conservés à l'École des mines étaient l'objet de recherches précises et que le personnel de la bibliothèque s'efforçait de mettre à la disposition de ses lecteurs des instruments de recherche adaptés. Quelques années plus tard, les travaux de catalogage s'attachèrent à des collections spécifiques pour répondre à l'accroissement des recherches constaté depuis la fin de la Seconde Guerre mondiale. En 1949, l'inventaire des 2000 périodiques conservés à la bibliothèque fut réalisé dans des fichiers kardex. Les fiches, rangées par ordre alphabétique, précisaient l'état détaillé de chaque collection ; un catalogue géographique vint compléter cet inventaire ${ }^{27}$. Les années 1940 furent également marquées par la constitution d'un catalogue spécifiquement consacré aux livres anciens. En 1941, ce fonds fut de nouveau inventorié et numéroté ${ }^{28}$, puis, entre 1950 et 1955 , le catalogue des auteurs anciens fut recopié à partir des registres, afin de disposer d'une connaissance plus précise de ce fonds qui n'était que partiellement répertorié dans le tout premier catalogue sur fiches dressé au cours des années 1840.

$\mathrm{Du}$ milieu du $19^{\mathrm{e}}$ au milieu du $20^{\mathrm{e}}$ siècle, la bibliothèque de l'École des mines s'est vue dotée de salles spécifiques et d'instruments de recherche censés donner corps à ses collections. Les catalogues sur fiches mis à la disposition des lecteurs dans une salle particulière apportèrent une existence nouvelle à la collection, les logiques d'indexation créèrent des parcours qui se détachaient de la topographie 
des rayonnages. Toutefois, l'absence d'archives qui auraient gardé la trace des demandes de consultations et d'emprunts ne permet malheureusement pas d'aller plus loin dans l'identification des usages développés au sein de la collection. Certains documents permettent en revanche de mesurer l'attachement symbolique des ingénieurs des Mines à leur bibliothèque.

\section{Enrichir une collection d'ouvrages scientifiques pour construire la mémoire du corps des Mines}

Parmi les missions actuelles de la bibliothèque figure la responsabilité du patrimoine écrit de l'École ${ }^{29}$. Progressivement, à partir du $19^{\mathrm{e}}$ siècle, la bibliothèque n'a plus seulement été vue comme une collection d'ouvrages scientifiques et techniques censés répondre aux besoins immédiats des professeurs et des élèves, elle est devenue le lieu où étaient versées les archives administratives de l'établissement, ou celles produites par d'anciens chercheurs de l'École ou par des élèves ${ }^{30}$, si bien que ses magasins abritent aujourd'hui 310 mètres linéaires de telles archives.

Outre les versements auxquels l'École a procédé, elle a, dès le $19^{\mathrm{e}}$ siècle, effectué des acquisitions à l'occasion de ventes de livres ou de papiers laissés par de prestigieux ingénieurs. Le premier registre de la bibliothèque indique par exemple que 35 ouvrages, pour la plupart en allemand, en anglais ou en italien, ont été achetés en 1841 lors de la vente des livres de Brochant de Villiers, ingénieur puis inspecteur général du corps des Mines, professeur de minéralogie et de géologie à l'École de Pesey, puis à l'École royale des mines de Paris ${ }^{31}$. Pour certains ensembles de manuscrits - le fonds Monnet ou le fonds Gillet-Laumont -, aucune source ne permet de déterminer les modalités de leur entrée dans les collections. Cependant, la manière dont les mémoires et récits de voyage du premier ou la correspondance du second sont présentés laisse penser que ces ensembles ont été vendus par un libraire. Le choix de l'École n'est pas anodin puisque Monnet fait partie des tout premiers inspecteurs des Mines nommés par le contrôle général en 1776 ; quant à Gillet-Laumont, d’abord nommé inspecteur des Mines en 1784, il a animé la direction collégiale de l'Agence puis du Conseil des mines jusqu'en 1810. L'acquisition par l'École de ces documents témoigne de la volonté d'en préserver la mémoire et de conserver des indices de l'ancienneté du corps.
En 1907, l'École fait l'acquisition du lot de manuscrits baptisé aujourd'hui "fonds Élie de Beaumont " ${ }^{22}$. Il s'agit essentiellement de lettres que l'un des auteurs de la carte géologique de France a échangées avec ses correspondants, de carnets de terrain, du brouillon de l'explication de la carte géologique de France, etc. Quelques années auparavant, Élie de Beaumont avait donné 167 ouvrages (plus de 300 volumes) de sa bibliothèque à l'École. La liste présente 100 titres d'ouvrages - périodiques et monographies - publiés en français, 46 en allemand, 15 en anglais, 6 en italien, enfin on y trouve une vingtaine de cartes ou d'atlas ${ }^{33}$. Il s'agit pour l'essentiel d'ouvrages publiés au $19^{\mathrm{e}}$ siècle, dont un certain nombre de tirés à part, peut-être envoyés par leurs auteurs respectifs à Élie de Beaumont ; plusieurs contributions majeures à la cartographie géologique européenne s'y trouvent aussi. Élie de Beaumont a vraisemblablement donnéà l'École les ouvrages, ou du moins une partie de ceux qui constituaient sa bibliothèque de travail personnelle. $\mathrm{Au}$ cours des dernières décennies du $19^{\mathrm{e}}$ siècle, certains ingénieurs prennent l'habitude de faire don d'une partie de leurs livres à la bibliothèque de l'École où ils ont été formés, voire où ils ont enseigné. En 1885, la bibliothèque reçoit ainsi 439 des ouvrages de Louis Le Chatelier ${ }^{34}$. Il s'agit pour l'essentiel d'ouvrages de travail qu'il conservait dans les locaux de l'École. D'autres lèguent une partie de leur bibliothèque personnelle, comme c'est le cas avec le don de la "bibliothèque géologique " de Lucien Cayeux, effectué par sa veuve en 1951. L'ampleur de ce don est telle - 1051 entrées figurent dans le registre où sont répertoriés les dons - que le personnel de la bibliothèque ne peut, à lui seul, cataloguer les ouvrages donnés et les intégrer à la collection. Il faut attendre une subvention obtenue en 1956 et 1957 pour que ce fonds rejoigne les collections de l'École.

Â partir du milieu du $19^{\text {e }}$ siècle, les dons deviennent de plus en plus fréquents. Ils sont souvent partiels et éclairent une partie de l'activité professionnelle du donateur : il peut s'agir par exemple de notes de cours prises par le défunt pendant sa scolarité à l'École, de carnets de terrain ou encore de minutes de cartes géologiques. Certains ingénieurs des Mines font le choix de léguer à la bibliothèque, à la fin de leur carrière, tout ou partie des travaux qu'ils ont publiés. C'est le cas d'Edmond Friedel, qui a été sous-directeur de l'École et qui, en juin 1943, annonce avoir rassemblé les notes qu'il a publiées sur les insectes et plantes fossiles de la potasse d'Alsace, pour en faire don à la bibliothèque.

À la même période, on voit se développer des dons de livres anciens, supposés renforcer le caractère patrimonial de la bibliothèque de l'École des mines. Daubrée, qui a dirigé l'établissement entre 1872 et 1884, a par exemple fait 


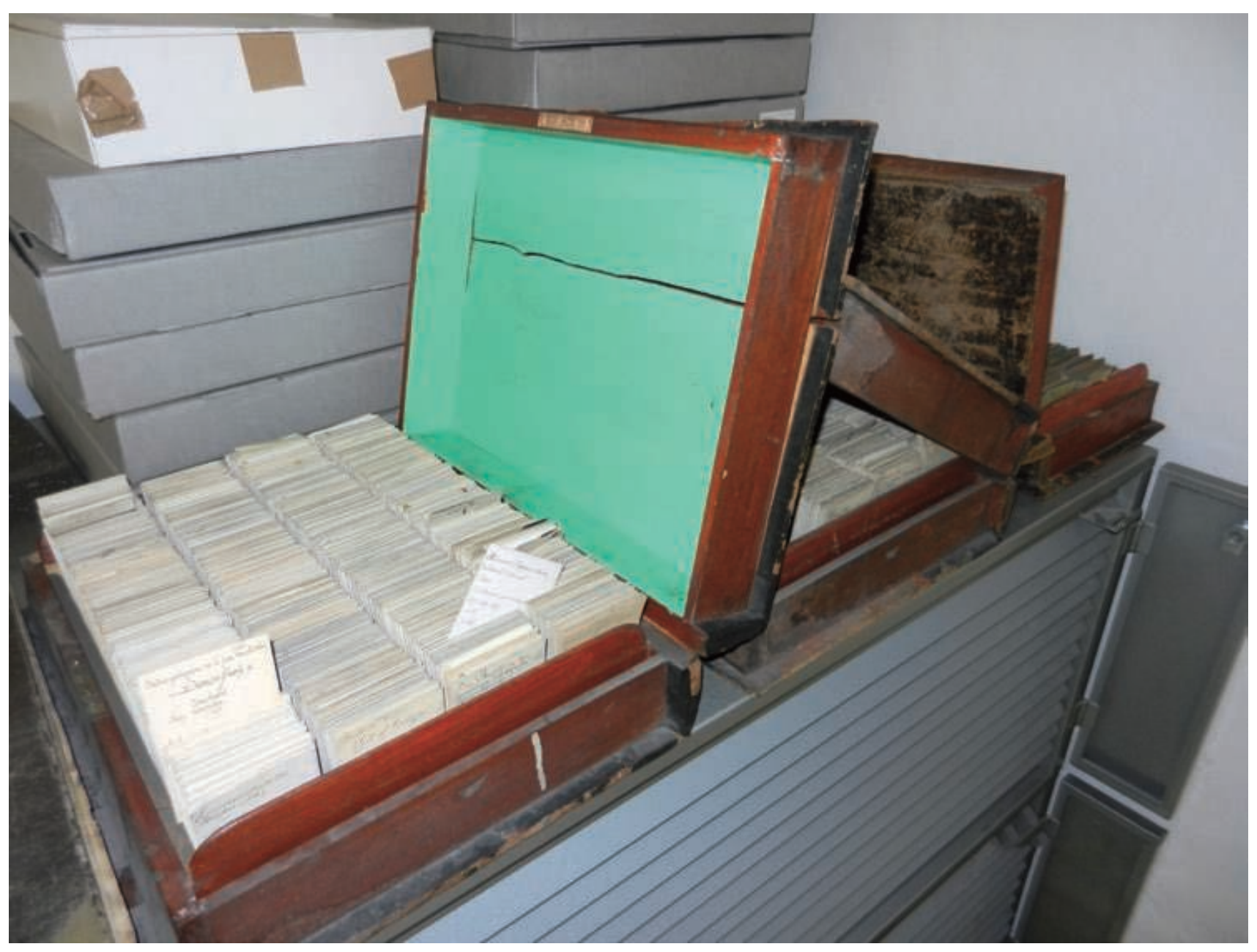

Premier catalogue sur fiches conservé dans les magasins de la bibliothèque de l'École des mines de Paris (coll. bibliothèque de l'École des mines de Paris) 
don de son exemplaire du Bergbüchlein (1505) et d'autres ouvrages anciens sur la mine et la métallurgie ${ }^{35}$. Un exdono identifie ses ouvrages. En 196o, la veuve de Maurice Pesson-Didion lègue à la bibliothèque 41 ouvrages anciens ayant appartenu à son époux. On trouve parmi eux : le De re metallica d'Agricola dans une réédition de 1912, les traités de Cardan, la première partie de L'Histoire entière des poissons de Rondelet ou encore le Grand lapidaire de Jean de Mandeville, dans des éditions du $16^{\text {e }}$ siècle, les œuvres de Palissy dans une réédition de 1888. Dans l'une des lettres de remerciements qu'il lui adressa, le directeur de la bibliothèque lui proposa d'exposer quelques-uns de ces volumes dans le hall de cette dernière, en donnant pour sujet à cette exposition "Bernard Palissy et son temps". De telles initiatives individuelles rejaillissent à la fois sur la notoriété du donateur et sur le prestige de l'École. Elles ont été complétées depuis quelques années par l'association $\mathrm{ABC}$ Mines qui se donne comme objectif de promouvoir la connaissance de l'École et de contribuer à l'enrichissement de ses collections. La liste des acquisitions effectuées depuis 1992 regroupe des ouvrages anciens, dont l'ancrage épistémologique questionne l'identité du corps des Mines.

Si les grands domaines de savoirs représentés dans la bibliothèque de l'École des mines ont évolué à mesure que les programmes d'enseignement ont été repensés et que la culture professionnelle des ingénieurs s'est transformée, ce n'est probablement pas l'évolution la plus significative de ce lieu de savoir qui, progressivement, s'est vu doté d'un personnel spécifique, de fonds de mieux en mieux identifiés et répertoriés. L'attachement symbolique des ingénieurs à cette bibliothèque à la vocation patrimoniale croissante - l'ouverture de la bibliothèque patrimoniale numériquele montre ${ }^{36}$ - éclaire la place tenue par la science dans la culture professionnelle des membres du corps. Le statut privilégié qu'occupent encore aujourd'hui la minéralogie et la géologie dans la valorisation des collections patrimoniales illustre bien l'attachement des ingénieurs des Mines à cette identité originelle du corps.

\section{Isabelle Laboulais}

\section{Notes}

1 - Si l'École royale des mines est créée en 1783 à l'hôtel de la Monnaie, il n'existe aucune continuité entre les collections de livres et de spécimens qui s'y trouvent et celles constituées à partir de 1794 pour les besoins des professeurs et des élèves des Mines. Sous l'Ancien Régime, les collections conservées à la Monnaie sont celles de Sage, titulaire de la chaire de minéralogie.

2 - " Ce lien communautaire dans la longue durée de la lignée ou dans le présent où s'actualise le cercle, se traduit dans les gestes et dans les savoir-faire, dans les codes d'interaction et les rythmes du quotidien aussi bien que dans les doctrines et les connaissances partagées " (Jacob, Christian, Le cercle et la lignée, in C. Jacob (dir.), Lieux de savoir. Espaces et communautés, Paris, Albin Michel, 2007, p. 132).

3- Bourdieu, Pierre, La Noblesse d'État, Paris, 1989, p. 101-102

4 - Nora, Pierre, Entre Mémoire et Histoire. La problématique des lieux, in P. Nora (dir.), Les lieux de mémoire, vol. 1, Paris, Gallimard, coll. Quarto, réédition 1997, p. 37

5 - En l'an VII, Clouet est chargé par le Conseil des mines de rédiger le " catalogue par ordre alphabétique des auteurs contenus ", en se conformant aux indications de l'instruction du ministre de l'Intérieur. Ce catalogue n'a malheureusement pas été conservé, il n'existe pas non plus d'inventaire global. Cf. bibliothèque de l'École des mines de Paris, $\mathrm{ms} 78, \mathrm{~V} 7 \mathrm{a}$, Lettre du Conseil des mines au citoyen Clouet, le 8 fructidor an VII (25 août 1799).

6- Bradley, Margaret M., An early science library and the provision of textbooks: the École polytechnique, 1794-1815, in Libri, t. 26, 1976, p. 166

7- Bibliothèque de l'École des mines de Paris (Bib. EMP), ms 78, If, Lettre du Conseil des mines de la République aux citoyens conservateurs des Dépôts littéraires de la Commission temporaire des arts, le 21 brumaire an IV (12 novembre 1795).

8 - Bib. EMP, ms 78, VII 2, "Copie de l'arrêté de la Commission des armes et poudres de la République une et indivisible du 6 floréal an III ".

9 - Bib. EMP, ms 78, Ik, Note de l'Agence des mines, Maison d'instruction, bibliothèque, s. $\mathrm{d}$.

10- Bib. EMP, ms 78, If, Lettre du Conseil des mines de la République aux citoyens conservateurs des Dépôts littéraires de la Commission temporaire des arts, le 21 brumaire an IV (12 novembre 1795).

11 - Bib. EMP, ms 78, IV 10, " Notice des livres extraits des dépôts nationaux, utiles et nécessaires à la Bibliothèque de la Maison d'instruction pour l'exploitation des mines de la République ».

12 - Laboulais, Isabelle, La Maison des mines. La genèse révolutionnaire d'un corps d’ingénieurs civils (1794-1814), Rennes, Presses universitaires de Rennes, 2012

13- Archives nationales, F 14 1053, Lettre de Schreiber au Conseil des Mines, Pesey, le 2 nivôse an XII (24 décembre 1803).

14-AN, F 14 1047, "Inventaire général et estimatif des immeubles, meubles et autres objets, existant dans les établissements dépendants de l'École pratique des mines du Mont Blanc, au 1er janvier 1813 ".

15 - Notice imprimée par le Conseil de perfectionnement de l'École, 1904.

16 - Dans les locaux occupés par l'École des ponts et chaussées entre la fin du $18^{e}$ et le début du $19^{e}$ siècle, il n'existe aucune pièce faisant office de bibliothèque ou de lieu d'étude pour les élèves. Voir Montel, Nathalie, Une revue des savoirs d'État. De la genèse à la fabrique des Annales des ponts et chaussées au XIX siècle, Habilitation à diriger des recherches, Université de Paris I, 2008, p. 534.

17 - Bib. EMP, ms 78, Ig 
18 - Bib. EMP, ms 78, V 3 et V 8, ensemble de fiches nominatives classées par ordre alphabétique et répertoriant tous les ouvrages empruntés par une même personne. Voir aussi ms $78, \mathrm{~V} 6$, Liste des ouvrages empruntés.

19 - AN, F 14 11059, " Rapport à Monsieur le directeur général ", le 23 juillet $1817, \mathrm{f}^{\circ} 1$

20 - Maisonneuve, Marie-Noëlle, Masson, Francine, L'École des mines : histoire de sa bibliothèque et de ses collections, in Arts et métiers du livre, mars-avril 2008, p. 30-43

21 - AN, F 14 11059, ibidem, $\mathrm{f}^{\circ} 10$

22- Catalogue des livres composant la bibliothèque de l'École royale polytechnique, Paris, Bachelier, 1840, 2 vol. ; Catalogue de la bibliothèque de l'École polytechnique, Paris, Gauthier-Villars, 1881.

23 - Catalogue des livres composant la bibliothèque de l'École nationale des ponts et chaussées, Paris, Imprimerie nationale, 1894

24-Bibliothèque de l'École nationale supérieure des mines : catalogue, Paris, Imprimerie nationale, 1899, 2 volumes

25- Masson, Francine, La bibliothèque de l'École des mines de Paris comme source de (re)connaissance d'un enseignement, in Documents pour l'histoire des techniques, $\mathrm{n}^{\circ} 16,2008$, p. 75

26- Masson, Francine, Per piacer di porle in lista. Réflexions désordonnées sur quelques catalogues de bibliothèques scientifiques au XIX eiècle, in Bulletin des bibliothèques de France, $\mathrm{n}^{\circ}$ 1, 2001, p. 33-36

27- Bib. EMP, Archives de la bibliothèque, Discours du directeur prononcé en juin 1949.

28- Quelques années plus tard, en 1948, le Département de la réserve a été créé.

29- Maisonneuve, Marie-Noëlle, Les sources manuscrites de l'histoire des mines à la Bibliothèque de l'École des mines de Paris, in Documents pour l'histoire des techniques, $\mathrm{n}^{\circ} 16$, décembre 2008, p. 67-73

30- Benoît, Serge, Maisonneuve, Marie-Noëlle, Un gisement iconographique de premier ordre : les relations de voyages d'élèves-ingénieurs à l'École nationale supérieure des mines de Paris, in Actes de la table ronde du groupe d'histoire des mines, Pierres et terre, $\mathrm{n}^{\circ} 33,1990, \mathrm{p} .109-132$

31 - Bib. EMP, Registre $n^{\circ} 1$, décembre 1841

32 - https:/|patrimoine.mines-paristech.fr|

33 - Bib. EMP, ms 78 VII, "Liste des ouvrages provenant de la bibliothèque de M. Élie de Beaumont et donnés à l’École des mines ".

34- Bib. EMP, ms 78 VII, "Liste des ouvrages donnés à la Bibliothèque de l'École des mines par M. Le Chatelier (H. L.), ingénieur des Mines ", 22 octobre 1882 .

35 - Maisonneuve, Marie-Noëlle, Masson, Francine, op. cit., p. 40

36 - https:/|patrimoine.mines-paristech.fr| 